03.1

\title{
Подавление неустойчивости течений с вращением
}

\author{
( Д.Ю. Жиленко, О.Э. Кривоносова \\ Институт механики Московского государственного университета им. М.В. Ломоносова, Москва, Россия \\ E-mail: jilenko@imec.msu.ru
}

Поступило в Редакцию 9 ноября 2021 г.

В окончательной редакции 16 декабря 2021 г.

Принято к публикации 22 декабря 2021 г.

\begin{abstract}
Экспериментально исследованы возможности управления неустойчивостью, развивающейся в виде бегущих азимутальных волн в сферическом течении Куэтта. Установлено, что воздействие на бегущие азимутальные волны периодической модуляцией скорости вращения внутренней сферы может приводить к их подавлению, при отключении модуляции неустойчивость в виде бегущих азимутальных волн восстанавливается, в том числе со сменой волнового числа. Обнаружена возможность длительного во времени сохранения устойчивого стационарного течения после окончания модуляции (не менее 50 min или 2000 оборотов внутренней сферы), что в 4 раза больше времени переходных процессов в стационарном течении.
\end{abstract}

Ключевые слова: геофизические течения с вращением, сферическое течение Куэтта, управление неустойчивостью.

DOI: 10.21883/PJTF.2022.06.52206.19073

Исследованию возможностей подавления гидродинамической неустойчивости течений посвящено много экспериментальных и теоретических работ. Так, например, ослабление неустойчивости в ламинарных пограничных слоях может происходить при силовом воздействии, создаваемом электрическими разрядами $[1,2]$. Стабилизация течения возможна под действием ускоренного сдвигового течения [3], поперечных осцилляций [4] или массообмена на границе течения [5], а также под действием магнитного [6] или электрического [7] полей. Поиск методов подавления неустойчивости в течениях с вращением интересен для технологических приложений, таких как выращивание кристаллов из жидких расплавов и растворов [8]. В этом случае гидродинамическая неустойчивость вызывает неоднородность фронта кристаллизации с образованием примесных полос роста, а подавление неустойчивости повышает однородность структуры монокристаллов [9]. В сферическом течении Куэтта, которое и является предметом исследования настоящей работы, периодическая во времени модуляция скорости вращения одной из сферических границ позволяет обеспечить частичное [10] или полное [11] подавление турбулентности, смещение величины предела устойчивости [12]. В последнем случае была показана возможность уменьшения интенсивности неустойчивых течений, но остается открытым вопрос о поведении течений после снятия управляющего воздействия. Прояснение этого вопроса и является целью настоящей работы.

Эксперименты по управлению неустойчивостью проводились в сферическом течении Куэтта, представляющем собой течение вязкой несжимаемой жидкости в зазоре между коаксиально расположенными сферами, которое формируется под действием вращения одной или обеих границ, радиусы внутренней и внешней сфер составляют $r_{1}=0.075 \mathrm{~m}$ и $r_{2}=0.15 \mathrm{~m}$ соответственно. Слой наполнялся силиконовым маслом с вязкостью $v \approx 5 \cdot 10^{-5} \mathrm{~m}^{2} / \mathrm{s}$ при температуре $22^{\circ} \mathrm{C}$, в масло для визуализации течения добавлена алюминиевая пудра. С целью стабилизации температуры сферы размещены в заполненном силиконовым маслом термостате. Температура рабочей жидкости в слое поддерживалась постоянной (с точностью не менее $\pm 0.05^{\circ} \mathrm{C}$ ) и контролировалась расположенным на экваторе внешней сферы датчиком. Измерения азимутальной компоненты скорости течения $u_{\varphi}$ лазерным анемометром проводились вблизи внешней сферы. Точка измерения удалена от плоскости экватора на расстояние $0.078 \mathrm{~m}$ и от оси вращения на $0.105 \mathrm{~m}$. В настоящей работе внешняя сфера неподвижна, скорость вращения внутренней сферы $\Omega_{1}(t)$ изменялась относительно средней скорости $\Omega_{10}$ с задаваемой системой управления амплитудой $A$ и частотой $f$

$$
\Omega_{1}(t)=\Omega_{10}(1+A \sin (2 \pi f t+\varphi)) .
$$

Вращение сфер регулируется цифровой системой управления с выделенным сигнальным процессором, которая обеспечивает сохранение как среднего значения угловой скорости с точностью $\pm 0.05 \%$, так и величин амплитуды и частоты. Мгновенные значения угловой скорости внутренней сферы $\Omega_{1}(t)$ вычисляются путем дифференцирования по времени сигналов фазы, поступающих с расположенного на валу датчика скорости вращения. Более подробное описание экспериментальной установки приведено в [13].

Эксперименты проводились следующим образом: сначала при $A=0$ квазистатически увеличивалась $\Omega_{10}$ до 

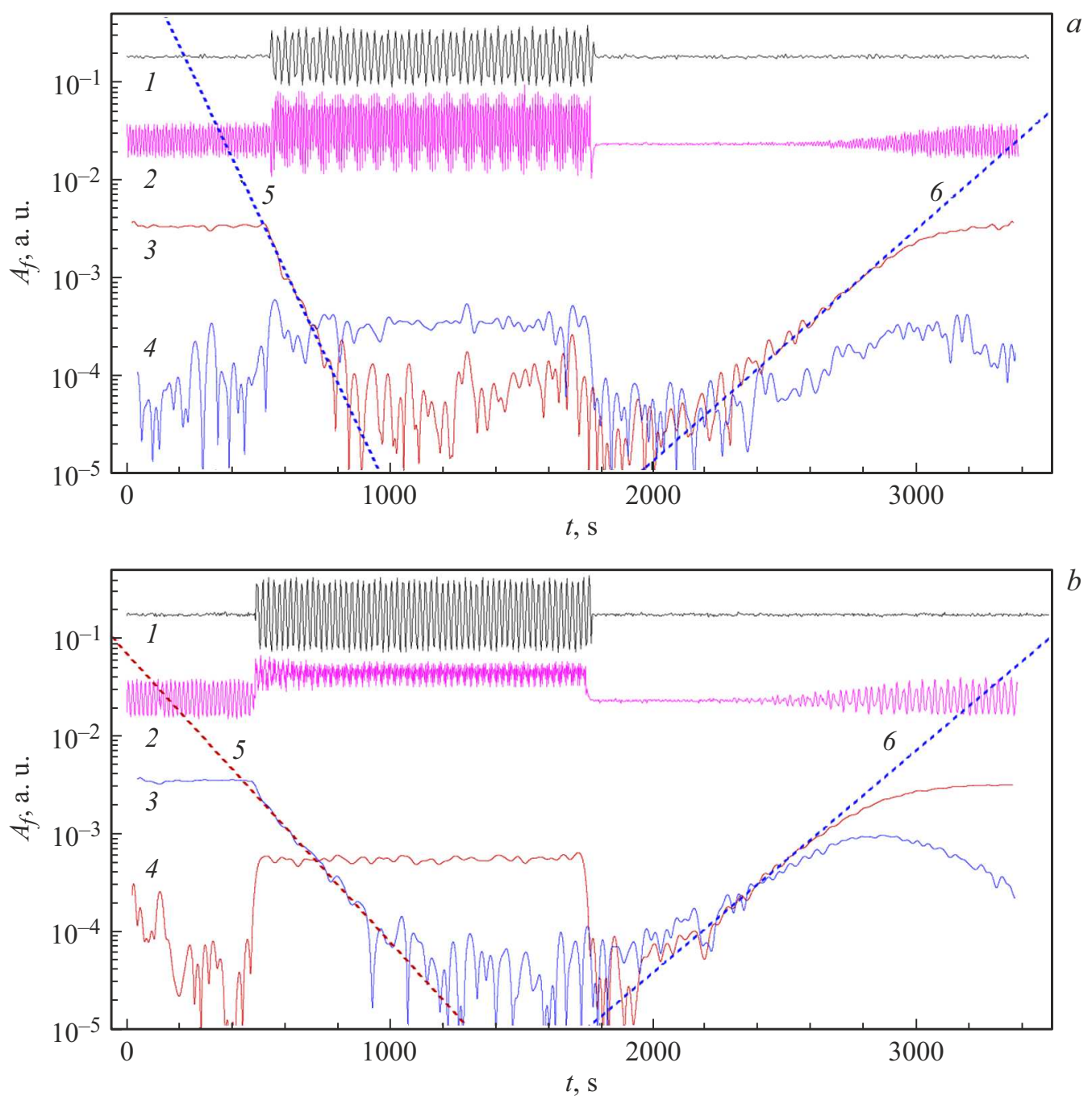

Рис. 1. Зависимости от времени $t: 1-$ скорости внутренней сферы $\Omega_{1}(t) / 2 \pi, 2-$ измеряемой скорости течения $u_{\varphi}(t), 3,4-$ амплитуд $A_{f}(t)$ начальной и вторичной мод соответственно, 5,6 - экспоненциальные аппроксимации при снижении и нарастании $A_{f}(t)$ соответственно. $a-A=0.306, f=0.1 \mathrm{~Hz}$, начальная мода $m=4, b-A=0.382, f=0.2 \mathrm{~Hz}$, начальная мода $m=3$. Для наглядности зависимости 1 и 2 показаны с увеличенным временны́м интервалом.

значения числа Рейнольдса

$$
\operatorname{Re}_{1}=\left(\Omega_{10} r_{1}^{2}\right) / v
$$

превышающего предел устойчивости течения. В рассматриваемом сферическом слое при стационарном вращении внутренней границы пределу устойчивости течения соответствует критическое значение числа Рейнольдса $\operatorname{Re}_{c}=460 \pm 2$ [13]. При выбранном значении $\operatorname{Re}_{1}$ (во всех представленных здесь экспериментах отношение $\operatorname{Re}_{1} / \operatorname{Re}_{c}=1.0046$ оставалось постоянным) формировалось неустойчивое нестационарное течение в виде бегущих азимутальных волн с волновыми числами $m=3$ или $m=4$. Волны распространяются в направлении вращения внутренней сферы с отставанием от нее [13]. C течением времени при $\mathrm{Re}_{1}=$ const после потери устойчивости сохраняется только одна мода, далее называемая начальной, соответственно вторую моду назовем вторичной. Выбор волнового числа $m$ определяется конкуренцией азимутальных мод 3 и 4 и зависит от начальных условий и уровня шума в сигнале скорости вращения $[13,14]$. Запись измерений $u_{\varphi}$ начиналась после потери течением устойчивости и определения волнового числа начальной моды (определялись по визуализации течения). Затем величина $A$ ступенчатым образом увеличивалась от нуля до требуемого значения, которое сохранялось постоянным в течение 16-20 min, после чего модуляция отключалась с возвращением к стационарному вращению внутренней границы.

В случае течений с начальной модой $m=3$ использовались частоты модуляции $f=0.2,0.25 \mathrm{~Hz}$, в случае $m=4-f=0.1,0.2,0.25,0.3 \mathrm{~Hz}$. Так же как и в [12,14], запись сигнала скорости использовалась для определения амплитуд колебаний $A_{f}$, соответствующих моде вторичного течения, с использованием преобразования 


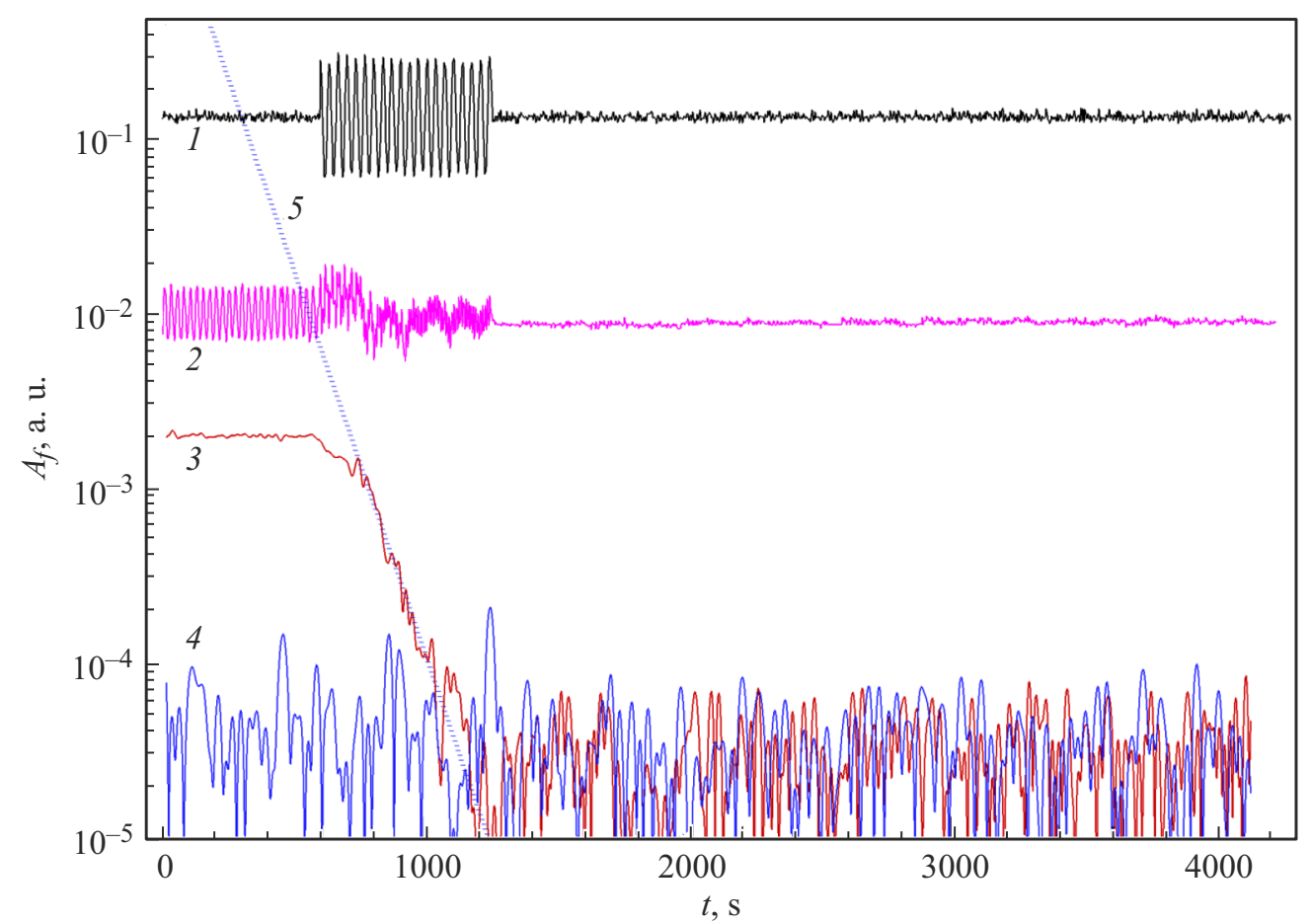

Рис. 2. Зависимости от времени $t$. Обозначение линий $1-5$ то же, что и на рис. $1 . A=0.184, f=0.25 \mathrm{~Hz}$, начальная мода $m=4$.

Гильберта (НТ):

$$
A_{f}=\left|u_{f}(t)+i \mathrm{HT}\left(u_{f}(t)\right)\right|
$$

где $u_{f}(t)$ - результат фильтрации сигнала скорости $u_{\varphi}(t)$ в полосе частот $f_{3} \pm \Delta f$ для $m=3$ и $f_{4} \pm \Delta f$ для $m=4, \Delta f=0.005-0.01 \mathrm{~Hz}$, частоты азимутальных мод $f_{3}=0.3-0.32 \mathrm{~Hz}$ и $f_{4}=0.4-0.43 \mathrm{~Hz}[12-14]$. Зависимости $\Omega_{1}(t) / 2 \pi, u_{\varphi}(t)$ и $A_{f}(t)$ представлены на рис. 1. Видно, что под действием модуляции в точке измерения изменяется средняя скорость течения, и начинается экспоненциальное затухание амплитуды начальных мод течения ( $m=4$ на рис. $1, a$, кривая $3 ; m=3$ на рис. $1, b$, кривая 3 ), что полностью соответствует полученным ранее в [12] результатам. Такое поведение начальных мод характерно при частотах модуляции $f=0.1-0.25 \mathrm{~Hz}$ и всех амплитудах модуляции $A$. При $f=0.3 \mathrm{~Hz}$ поведение начальных мод изменяется: при малых $A$ наблюдается возрастание амплитуды начальной моды $A_{f}$, а при увеличении $A-$ ее уменьшение.

В то же время под влиянием модуляции могут увеличиваться амплитуды вторичных мод $(m=3$ на рис. $1, a$, кривая $4 ; m=4$ на рис. $1, b$, кривая 4$)$, которые пренебрежимо малы в случае стационарного вращения. Во время действия модуляции амплитуды вторичных мод могут значительно превышать амплитуды начальных мод (рис. 1, $b$, кривые 3,4 ). Такое поведение вторичной моды не зависит от волнового числа начальной моды при $f=0.1,0.2 \mathrm{~Hz}$, при увеличении $f$ амплитуда вторичной моды практически не изменяется. После окончания действия модуляции амплитуды конкурирующих мод сравниваются и приближаются к величинам $A_{f}$ вторичных мод до начала модуляции: происходит затухание либо обеих мод (рис. 1, $a$ ), либо только вторичной моды (рис. $1, b)$. После окончания переходных процессов в течении, вызванных возвращением к стационарному вращению (не более $600 \mathrm{~s}$ ), обе моды начинают экспоненциально возрастать. Амплитуда одной из них выходит со временем на стационарное значение, тогда как амплитуда другой переходит через максимум и начинает уменьшаться. Аналогичное поведение конкурирующих мод экспериментально наблюдалось в случае действия ускорения и шума на течение [14]. В тех случаях, когда начальной является мода $m=3$ (рис. 1,b), выравнивание амплитуд мод после отключения модуляции и окончания переходных процессов может приводить к тому, что инкремент нарастания моды $m=4 \lambda_{i 4}$ становится выше аналогичной величины $\lambda_{i 3}$ моды $m=3$, что наблюдается также и при стационарном вращении $[13,14]$. В результате происходит смена волнового числа без изменения средней скорости вращения (рис. $1, b$, кривая 1 ). Если начальной является мода $m=4$, то она всегда остается доминантной после окончания модуляции.

С увеличением амплитуды модуляции поведение течения после окончания модуляции может измениться (рис. 2). Под действием модуляции амплитуда начальной моды $m=4$ уменьшается и сравнивается с амплитудой вторичной моды. После окончания модуляции амплитуды обеих мод длительное время (около 3000 s или 2000 оборотов внутренней сферы) не возрастают, и течение остается устойчивым. Необходимо отметить, что такое 

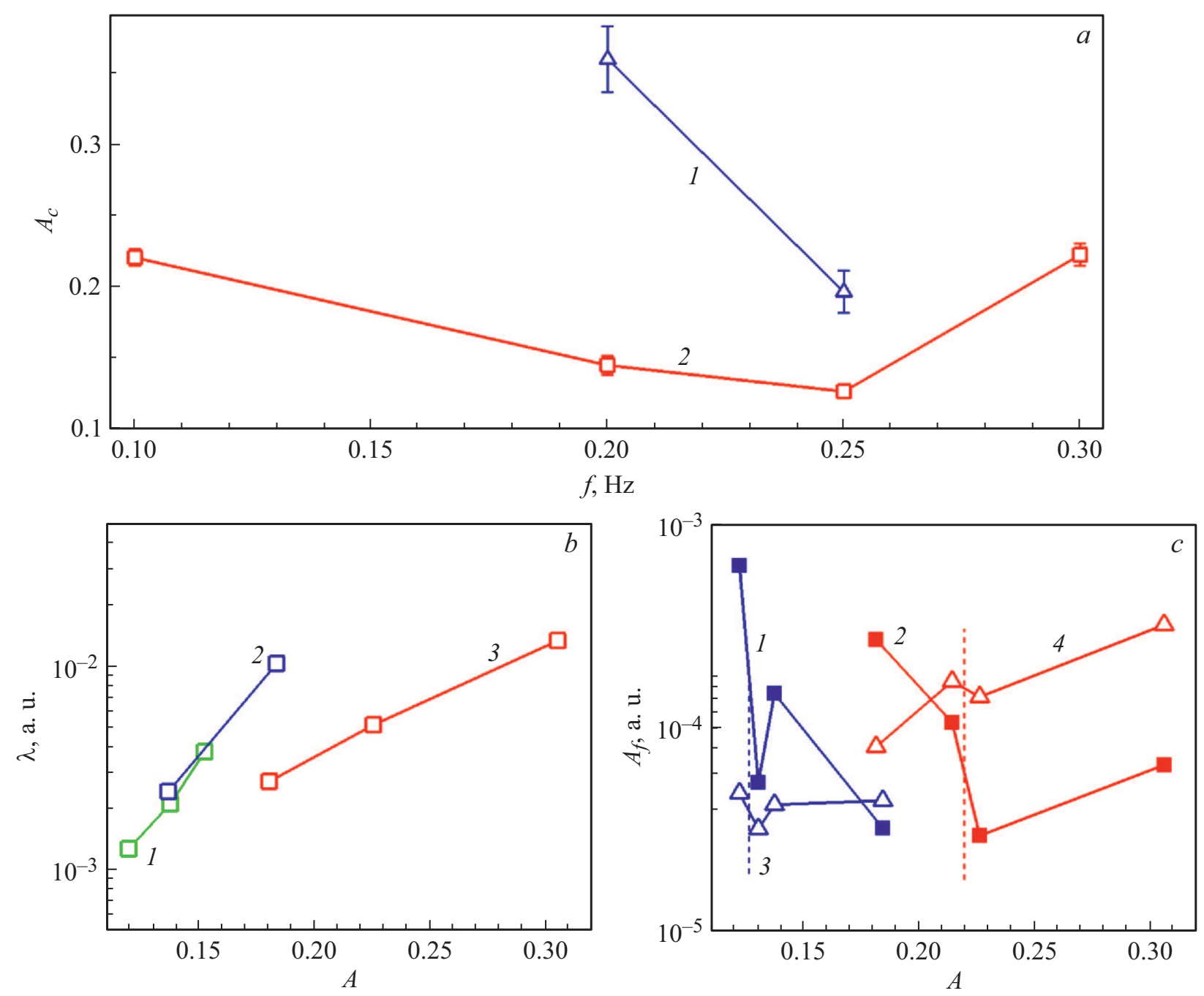

Рис. 3. $a-$ - зависимости критической величины амплитуды модуляции $A_{c}$ от частоты $f$ при начальной моде $m=3(1)$ и $4(2)$. Вертикальные отрезки соответствуют величине шага ступенчатого изменения $A$, при котором наблюдалось длительное сохранение устойчивости. $b$ - зависимости от амплитуды $A$ декремента затухания $\lambda$ начальной моды $m=4$ при $f=0.2(1), 0.25(2)$ и $0.1 \mathrm{~Hz}(3) . c$ - зависимости средней величины $A_{f}(t)$ начальной моды $m=4(1,2)$ и вторичной моды $m=3(3,4)$ от амплитуды модуляции $A$ при $f=0.25(1,3)$ и $0.1 \mathrm{~Hz}(2,4)$. Штриховые вертикальные линии соответствуют $A_{c}$.

длительное сохранение течением устойчивости значительно превышает времена переходных процессов при квазистатическом ступенчатом изменении $\Omega_{1}$. При продолжении эксперимента через более продолжительный интервал времени (5000 s в случае, представленном на рис. 2) в течении неизбежно развивается неустойчивость.

На рис. 3, a показана зависимость от частоты модуляции $f$ критического значения амплитуды модуляции $A_{c}$, превышение которой ведет к сохранению течением устойчивости после окончания модуляции более $900 \mathrm{~s}$, что в 1.5 раза выше времени переходных процессов. Видно, что в случае начальной моды $m=3 A_{c}$ значительно выше, чем в случае $m=4$.

Рассмотрим подробнее, как именно воздействует возрастание амплитуды модуляции $A$ на течение. Прежде всего возрастание $A$ ведет к увеличению декрементов экспоненциального затухания амплитуд начальных мод (рис. $3, b)$ и возрастанию амплитуд вторичных мод. Соотношение средних по времени амплитуд начальной $(m=4)$ и вторичной $(m=3)$ мод во время действия модуляции показано на рис. 3,c. Видно, что с приближением $A$ к критическим значениям (показаны вертикальными штриховыми линиями) происходит первое сближение величин амплитуд начальной и вторичной мод, и здесь же наблюдаются локальные экстремумы на зависимости $A_{f}$ от $A$. Таким образом, как видно из pис. 3, $c$, при $A>A_{c}$ амплитуды вторичной моды $m=3$ либо выше амплитуд начальной моды $m=4$ (которая, напомним, является доминирующей при стационарном вращении), либо очень близки к ней. И именно в этой области $A>A_{c}$ наблюдается длительное сохранение устойчивости течений после отключения модуляции скорости вращения. 
Из представленных данных следует вывод, что именно взаимодействие конкурирующих и близких по амплитуде азимутальных мод является причиной обнаруженного в настоящей работе явления - длительного во времени сохранения устойчивости течений после снятия управляющего воздействия, подавляющего неустойчивость.

Изменение времени развития неустойчивости и ранее встречалось в экспериментах в рассматриваемом сферическом слое. Так, время взаимодействия мод может увеличиваться при увеличении амплитуды вносимого в течение шума [14] и изменяться при варьировании начальных условий в случае вращения внутренней сферы с постоянным по величине ускорением [15]. В настоящей работе увеличение времени взаимодействия происходит под влиянием знакопеременного ускорения, вызывающего существенное изменение соотношения амплитуд мод по сравнению со случаем стационарного вращения. Знакопеременное ускорение может приводить и к смене волнового числа $m=3$ на $m=4$ при восстановлении неустойчивости после возвращения к стационарному вращению.

\section{Финансирование работы}

Работа выполнена при частичной финансовой поддержке Российского фонда фундаментальных исследований (проект № 19-05-00028).

\section{Конфликт интересов}

Авторы заявляют, что у них нет конфликта интересов.

\section{Список литературы}

[1] С.А. Баранов, М.Д. Гамируллин, А.Ф. Киселев, А.П. Курячий, Д.С. Сбоев, С.Н. Толкачев, С.Л. Чернышев, ДАН, 488 (2), 147 (2019). DOI: 10.31857/S0869-56524882147-15 [S.A. Baranov, M.D. Gamirullin, A.P. Kiselev, A.P. Kuryachii, D.S. Sboev, S.N. Tolkachev, S.L. Chernyshev, Dokl. Phys., 64 (9), 365 (2019). DOI: 10.1134/S1028335819090039].

[2] K.-S. Choi, J.-H. Kim, Exp. Fluids, 59, 159 (2018). DOI: $10.1007 / \mathrm{s} 00348-018-2609-\mathrm{x}$

[3] Е.Е. Мешков, Р.В. Мокрецов, И.Р. Смагин, Изв. РАН. Механика жидкости и газа, № 5, 3 (2019). DOI: $\quad 10.1134 / \mathrm{S} 0568528119050074$ [E.E. Meshkov, R.V. Mokretsov, L.R. Smagin, Fluid Dyn., 54 (5), 591 (2019). DOI: 10.1134/S0015462819050070].

[4] S.M.E. Rabin, C.P. Caulfield, R.R. Kerswell, J. Fluid Mech., 738, R1 (2014). DOI: $10.1017 / \mathrm{jfm} .2013 .601$

[5] A. Kukala, S.J. Biringen, J. Fluid Mech., 738, 105 (2014). DOI: $10.1017 / \mathrm{jfm} .2013 .532$

[6] H.-H. Zhang, C. Zheng, N. Aubry, W.-T. Wu, Z.-H. Chen, Phys. Fluids, 32, 116104 (2020). DOI: 10.1063/5.0021980

[7] Н.М. Зубарев, Е.А. Кочурин, Письма в ЖЭТФ, 104 (4), 277 (2016). DOI: 10.7868/S0370274X16160128 [N.M. Zubarev, E.A. Kochurin, JETP Lett., 104 (4), 275 (2016). DOI: 10.1134/S0021364016160153].

[8] А.Я. Нашельский, Производство полупроводниковых материалов (Металлургия, М., 1989).
[9] Н.А. Верезуб, А.И. Простомолотов, И.В. Фрязинов, Кристаллография, 40 (6), 1056 (1995).

[10] Д.Ю. Жиленко, О.Э. Кривоносова, ЖТФ, 89 (7), 998 (2019). DOI: 10.21883/JTF.2019.07.47786.391-18 [D.Yu. Zhilenko, O.E. Krivonosova, Tech. Phys., 64 (7), 933 (2019). DOI: 10.1134/S1063784219070302].

[11] Д.Ю. Жиленко, О.Э. Кривоносова, Письма в ЖТФ, 45 (17), 20 (2019). DOI: 10.21883/PJTF.2019.17.48218.17740 [D.Yu. Zhilenko, O.E. Krivonosova, Tech. Phys. Lett., 45 (9), 870 (2019). DOI: 10.1134/S1063785019090141].

[12] Д.Ю. Жиленко, О.Э. Кривоносова, Письма в ЖТФ, 46 (12), 22 (2020). DOI: 10.21883/PJTF.2020.12.49522.18295 [D.Yu. Zhilenko, O.E. Krivonosova, Tech. Phys. Lett., 46 (6), 591 (2020). DOI: 10.1134/S1063785020060292].

[13] Ю.Н. Беляев, И.М. Яворская, Течения вязкой жидкости во вращающихся сферических слоях и их устойчивость. Итоги науки и техники. Сер. Механика жидкости и газа (ВИНИТИ, М., 1980), т. 15, с. 3-80.

[14] D. Zhilenko, O. Krivonosova, M. Gritsevich, P. Read, Chaos, 28, 053110 (2018). DOI: 10.1063/1.5011349

[15] Д.Ю. Жиленко, О.Э. Кривоносова, ДАН, 415 (6), 754 (2007). [D.Y. Zhilenko, O.E. Krivonosova, Dokl. Phys., 52 (8), 453 (2007). DOI: 10.1134/S1028335807080125]. 